\title{
Twitter Controls the Household Heating System
}

\author{
Nalin Asanka Gamagedara Arachchilage \\ Department of Computer Science \\ University of Oxford \\ Wolfson Building \\ Parks Road, Oxford
}

\author{
Stanislao Lauria, Steve Love \\ School of Information \\ Systems, Computing and Mathematics \\ Brunel University \\ Uxbridge, Middlesex
}

\begin{abstract}
The home is composed of many different devices, services and related technologies. These have a lack of communication with one another. A challenge within domestic central heating system is being able to exploit the functionality and offer a common means of monitoring and controlling, either locally or remotely. This paper introduces a domestic central heating system design for householders to control it through twitter, either locally or remotely. We believe by enabling householders to monitor and control domestic central heating system could contribute to make a smart home.
\end{abstract}

\section{Introduction}

One of the key challenges faced by the UK Government is to secure the increasing demand of energy supplies $[1,2]$. The government argues that securing energy supplies enable to save every sector of the economy. Therefore, tackling the heating demand is significantly vital because heating (both domestic and nondomestic) accounts for the largest single proportion of the UK's final energy demand at approximately 49 percent $[9,10]$. Furthermore, the government reported that households alone contribute 27 percent of UK carbon emissions with almost 75 percent of these domestic emissions happening from space and water heating $[9,10]$.

Information and Communication Technology (ICT) has become a backbone of today's society enabling not only a major impact on people's professional and private lives, but has also become one of the most significant drivers of economic growth. In fact, ICT also consumes energy, but most importantly it helps to conserve energy. Conventionally, ICT has done so by optimizing the performance of energy-using systems and processes in industry and commerce. For example, with the advancement of "smart" technology in the ubiquitous computing field, further ways of reducing growing levels of domestic energy consumption are now emerging [7]. Therefore, the appropriate design of technical systems to manage household heating systems could benefit to secure the increasing demand of energy supplies.
The design of central heating systems for the domestic environment has attracted increasing interest in the human factors discipline over the recent years [2, 3, and 4]. It is crucial that the system is designed to be as simple as possible to live with, offering easy way to control and automate the home. If the home central heating system is overly complex, the average householder is unlikely to use it [5]. This is mainly because the average resident, friends and family may have little technical experience of using the central heating system. Therefore, the system must be designed for householders so as not to require any technical background knowledge.

\section{Related work}

Residential thermostats have been a significant element in controlling domestic heating and cooling systems for over sixty years [6]. During this period, the consumer expectations regarding the quality of the central heating system have dramatically increased since thermostats are usually marketed as "energy saving". Therefore, consumers typically justify their purchase with this goal in mind. However, Caird and Roy stated that often-residential thermostats have been designed and installed without taking a sufficient account of user requirements and usability [2]. This might cause wasting energy instead of saving it. The design of operational heating systems paying particular attention to their usability needs to be concerned [3].

Dale and Crawshaw have stressed that the poor usability of thermostats and the need to improve their ergonomics almost thirty years ago [12]. They also stated, "it is easy to blame thermostat users for stupidity, but is slowly being realized that the problem of efficiency in practice properly belongs to the engineers or the system designers". Nevertheless, this issue is still problematic [1, 6, 7 and 11]. A "good" thermostat design is not only usable but also useful in a way that is cost-effective and compatible with existing equipment. Peffer et al. [11] suggested that further research is needed to design thermostats that can provide more comfortable and economical indoor environments. Furthermore, they highlighted 
that the thermostat is only one of many devices where human interaction plays a significant role in energy consumption. Arguably, a good programmable and controllable thermostat would facilitate energy-saving behavior and also provide comfort and convenience for the people using it. The ability to remotely program and control the device in a user friendly way is one aspect considered here to tackle this issue. To facilitate the control the interaction should be as natural as possible. Hence the use of social media tools such as Twitter is considered here as a communication protocol.

Twitter has resulted in a large amount of contributions around multiple topics. For example, Jansen, et al. [13] revealed that Twitter is a key tool for the customer word of mouth communications and marketing works. It can also be a worthwhile platform for mining public sentiment and reaction around popular events due to its extensive and diverse followers [14]. Furthermore, Twitter can be beneficial even in enterprise micro blogging activities such as coordinating loosely related individual tasks [15]. However, according to our best knowledge, Twitter has still not been used as a new communication protocol (to control remote devices) in the field of ubiquitous computing.

Literature revealed that some projects have used social networks such as Twitter and Facebook as a platform for energy efficiency activities, however, have not still gained much attention [7]. In 2012, the guardian reported that the UK is the fourth largest country for Twitter users in the world, with 80 percent accessing it with mobile phones [8]. It can increase exponentially in the near future due to pervasiveness of the information and communication technology. Therefore, this research focuses on designing a domestic central heating system for householders to control it through twitter. The novelty in this proposed design approach is the use of twitter as a new communication protocol where households are able to control their thermostats using remote devices such as smart phones, tablet PC, pages, and PDAs.

British Gas has recently introduced a remote heating control system, which is used to control households' central heating virtually anywhere and anytime from their smartphone or online [16]. Furthermore, they emphasize that it's simple to use and allows you to turn your heating on and off, set your heating schedule and adjust the temperature. However, we still argue that the proposed approach has a greater flexibility due to a number of reasons. First, it uses the communication protocol, therefore no need to be linked to any apps, accounts or profiles. Furthermore, the protocol has the ability to use twitter as two-way communication media rather than one-way. For example, sending tweets from human to thermostat and vice-versa. This might help the user to better interact with the thermostat in the dialog management aspect. Second, the protocol has an enormous potential in its ability to control the thermostat due to the possibility to "build" more complex functionality. Nevertheless, it can be argued that our proposed system provides simple, yet a rich set of user experience in controlling thermostat due to its well-developed twitter experience in the UK [8].

\section{Domestic central heating system design}

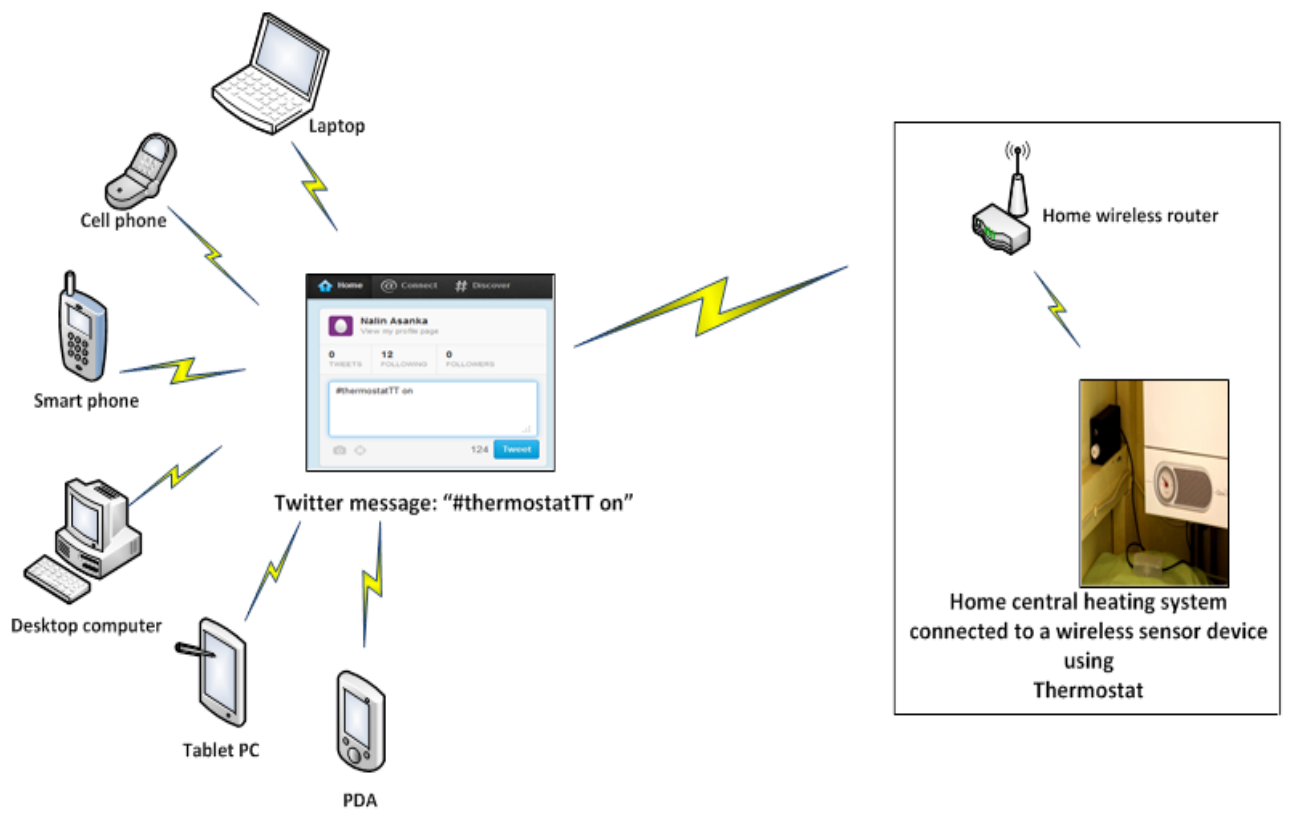

Figure 1. Domestic central heating systems architecture 
Twitter is an online social networking service that enables its users broadcast text-based messages of up to 140 characters, known as "tweets". The user can access to twitter using her/his laptop, desktop, or any hand-held device such as smart phone, PDA, and Tablet PC connected to the Internet. Thermostat is programmed in java to connect with the domestic central heating system (Fig. 3). The central heating system in the domestic environment connects to a wireless sensor device through a thermostat, which is used to keep on receiving signals from the wireless router (Fig. 3). Generally speaking, Internet data comes into the router from the domestic telephone line.

The user broadcasts a message using the twitter. The message should consist of a programmer-defined identifier (this could be keyword such as anhashtag such as \#thermostatTT) along with an instruction message (e.g. on or off). For example, when the user wants to turn off the domestic central heating system using his smart phone remotely, the twitter message looks like "\#thermostatTT off". The domestic thermostat is programmed only to execute the instruction message coming along with the "\#thermostatTT" keyword. A minimal architecture could include three instruction messages such as on, off and check. "\#thermostatTT check" is used to check whether or not heater switched on. "thermostatTT on" is used to tell the heater to switch on. "\#thermostatTT off" is used to tell the heater to switch off.

A java program code snippet can be written using "Thermostat" class in order to control the domestic central heating system as follows (Fig. 2 and 3).

intlowerLimit, upperLimit

boolean on

// true if $>=$ upperLimit, false if $<=$ lowerLimit

public Boolean is On() // Check whether or not the heater switched on

public void update( int reading) if !on \& reading > = upperLimit then set on and tell heater to switch off if on \& reading < = lowerLimit then set off and tell heater to switch on

Figure 2. Thermostat "tells" Heater to switch on/off

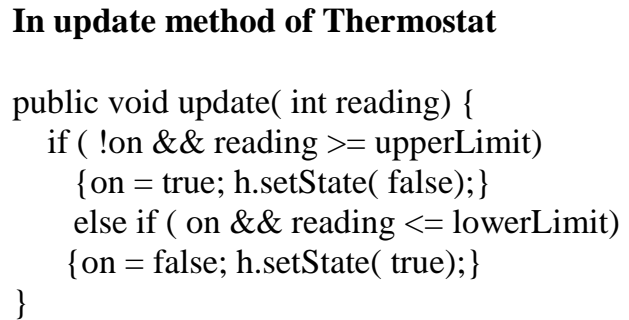

Need to connect Heater to Thermostat

Heater h; // new property added

public void setHeater( Heater h) \{ heater this.h $=\mathrm{h}$; \}

Figure 3. Thermostat "tells" Heater to switch on/off using a java code snippet

\section{Conclusions and Future Work}

This research focuses on presenting some initial aspects of defining a paradigm based on using social media protocols such as twitter to control a home central heating system for householders. This is motivated by the continuous growing of popularity of these tools. In turn this let us believe that users perceive these as natural way to communicate and interact. The paradigm is based on the idea that the user broadcasts a twitter message along with a key word. The wireless sensor connected to the thermostat keeps on receiving signal from the home wireless Internet router. Though the thermostat keep receiving all broadcast messages coming though twitter, it only listen and execute the message coming along with a unique identifier. Some aspects need to investigate further. For example, there might be a security implication for this research. The keyword along with the message is easily visible to twitter followers. In a simplified paradigm it is possible to assume no one follows the particular twitter account, therefore, it would be difficult to monitor the broadcasted messages. However, future research work can be concerned to address this issue. For example, the identifier (in the example discussed above the keyword "\#thermostatTT") can be encrypted by ADE 265 bit (Advanced Encryption Standard) before broadcasting the twitter message. In addition, as future research we attempt to implement the proposed domestic central heating system and observe how the user perception of using it to cut down unnecessary cost in the home use. Therefore, we believe that providing usable technology for householders to control their domestic central heating system will contribute to secure the increasing demand of energy supplies. 


\section{References}

[1]HM Government (2009) The UK Low Carbon Transition Plan. National Strategy for Climate and Energy, http://centralcontent.fco.gov.uk/centralcontent/campaigns/actoncopenhagen/resources/en/pdf/DECC-Low-CarbonTransition-Plan. (1 March 2013).

[2]Caird, S. \& Roy, R. (2010) Adoption and use of household micro generation heat technologies. Low Carbon Economy, 1(2), pp. 61-70. http://www.scirp.org/journal/lce. (12 September 2012).

[3]Sauer, J., Wastell, D. G. \&Schmeink, C. (2009) Designing for the home: A comparative study of support aids for central heating systems. Applied Ergonomics, 40, $165 \mathrm{e} 74$.

[4] Stanton, N. A. (1998) Human Factors in Consumer Products. Taylor \& Francis, London.

[5]Maternaghan, C. \& Turner, K. J. (2011) Programming Home Care. In Wolters, M. K., Turner, K. J. and Lakany, H. editors, Proc. Advances in Techniques and Technologies Assisting Care at Home. IEEE Computer Society, Los Alamitos, California, USA, May 2011, pp. 5.1-5.7.

[6] Meier, A. K., Aragon, C., Pefferm T., Perry, D. \&Pritoni, M. (2011) Usability of residential thermostats: preliminary investigations. Building and Environment, 46, 1891e8.

[7] Mattern, F., Staake, T. \& Weiss, M. (2010) ICT for Green - How Computers Can Help Us to Conserve Energy. Proc. e-Energy, ACM, New York, pp. 1-10.

[8] Arthur, C. (2012) The guardian, http://www.guardian.co.uk/technology/2012/may/15/ twitter-uk-users-10m. (14 September 2012).

[9] DTI (2007) Meeting the Energy Challenge, a White Paper on Energy. Department of Trade and Industry, London, http://www.officialdocuments.gov.uk/document/cm71/7124/7124.pdf. (1 March 213).

[10] HM Government (2009) The UK Renewable Energy Strategy: $\mathrm{Cm}$ 7686, Stationery Office, http://www.decc.gov.uk/assets/decc/what $\% 20$ we $\% 20$ do/uk\%20energy\%20supply/energy\%20mix/renewab le\%20energy/renewable\%20energy\%20strategy/1_2 0090717120647_e_@@_theukrenewableenergystrat egy2009.pdf. (12 September 2012).

[11] Peffer, T., Pritoni, M., Meier, A., Aragon, C.,
\&Perry, D. (2011). How people use thermostats in homes: A review. Building and Environment, 46 (12), pp. 2529-2541.

[12] Dale, H. C. A. \&Crawshaw, C. M. (1983) Ergonomic aspects of heating controls. Building Services Engineering Research and Technology, 4:22e5.

[13] Jansen, B. J., Zhang, M., Sobel, K. \&Chowdury, A. (2009) Twitter Power: Tweets as Electronic Word of Mouth. Journal of the American Society for Information Science and Technology, 60 (11), pp. 2169-2188.

[14] Thelwall, M., Buckley, K. \&Paltoglou, G. (2011) Sentiment in Twitter Events. Journal of the American Society for Information Science and Technology, 62(2), pp. 406-418.

[15] Riemer, K., Altenhofen, A. \& Richter, A. (2011) What Are You Doing? Enterprise Microblogging as Context Building.19th European Conference on Information Systems, Helsinki, Finland.

[16] British Gas (2013) Remote Heating Control, http://www.britishgas.co.uk/products-andservices/remote-heating-control.html. (3 March 2013). 